\title{
Resurrecting the future of strategy consulting
}

\section{Franz Liebl}

The road towards a successful and workable strategy is strewn with a host of obstacles. This does not only include the many strategy papers devised by consultants which are quietly shelved by management and never acted upon. Even strategy processes which are ostensibly based on the principle of participation and which involve the relevant stakeholders also have their inherent weaknesses.

The conclusions that can be drawn from this history of failure may be formulated in this way: first, organizations are evidently incapable of implementing strategies which are not in tune with their identity, however attractive and well thought out those strategies might appear to the impartial observer. Second, unless these identity issues are clarified at the very outset of strategy development, they crop up uncontrolled and often invisible during the strategy making process, say, for example, in the way people think about the future developments likely to face the company. Third, tedious strategy processes which seriously hamper the creative development of novel strategic options are the inevitable outcome, not to mention the (intended and unintended) distortions in future scenarios that result from these omissions.

Two fundamental questions have to be asked. First, what role does identity play in the different phases of the strategy making process? And, second, can we unleash the power of identities by making them transparent and by ensuring a proper alignment? What, therefore, are the creative potentials of a strategy process which makes sufficient allowance for identity, and what is the procedure for reaching this desirable goal?

With these questions Olaf Rughase has addressed a field that is highly significant for strategic success but where there has been surprisingly little research hitherto. Hence, his book contains some pathbreaking ideas which will impress both the practitioner and the academic. All the more so as Olaf Rughase - unlike many others writing on strategic management topics does not proceed ahistorically. Particularly for those who have followed the development of strategic management over the last 20 years and more, it is 
fascinating to see how Rughase extracts potentials for his central question from classic works by authors such as Ackoff, Abell and Andrews, which clearly have not been given the recognition they deserve in the scholarly discussion or which have inexplicably fallen into oblivion.

What Olaf Rughase's brilliant book conveys is the indissoluble alliance between strategy content and strategy process - a realization which, as I see it, will very much be the shape of things to come in strategy consulting. The future will not belong to the classic, content-driven 'recommendations' which, we're told, just have to be implemented by a top-management team 'with a strong hand'; nor will it be sufficient simply to 'irritate' an organization systemically in order to bring a viable strategy about. Olaf Rughase's answer here is closer to strategic practice because it is more complex. He highlights two issues pertaining to the relation between content and process which have to be addressed simultaneously. First, how must a strategy process be designed in order to be able to generate goaloriented and innovative contents? And second, what contents have to be fed into strategy making in order to fuel the process? It is only when both these aspects are taken into account that the preconditions are in place for strategic innovation which (at the same time) has a realistic chance of being implemented. 